Ann. Biol. anim. Bioch. Biophys., 1979, 19 (6), 1789-1799.

\title{
Effets du Solanum malacoxylon et du phosphore alimentaire chez le poulet en acidose métabolique chronique
}

\author{
par B. SAUVEUR \\ avec la collaboration technique de Huguette ANTOINE
}

Station de Recherches avicoles, I. N. R. A. Nouzilly 37380 Monnaie (France).

Summary. Effects of Solanum malacoxylon and dietary phosphorus on acidotic chicks.

Twelve groups of 52 day-old male chicks were submitted to the following factorially combined treatments : metabolic acidosis or not (induced by 2 p. 100 of $\mathrm{NH}_{4} \mathrm{Cl}$ in the dief), dietary inorganic phosphorus equal to 0.40 (normal level) or $0.65 \mathrm{p}$. 100 , allowance of vitamin $D_{3}$ only (1 $500 \mathrm{lU} / \mathrm{kg}$ of food), Solanum malacoxylon (SM) only (1.5 g of dry leaves $\left./ \mathrm{kg}\right)$ or a mixture of $D_{3}+S M\left(750 \mathrm{lU}\right.$ of $D_{3}+0.8 \mathrm{~g}$ of $\left.S M / \mathrm{kg}\right)$. Due to the severity of the acidosis, the six acidotic groups were studied for one week only. In non-acidotic birds, SM enabled normal body weight and ash percentage of the tibia up to 4 weeks of age, but induced a small decrease in plasma $\mathrm{Ca}$ level $\left(\mathrm{Ca}_{\mathrm{p}}\right)$. The increase of dietary phosphorus also decreased the $\mathrm{Ca}_{\mathrm{p}}$ but increased the tibia ash content. Induced metabolic acidosis hardly modified the $\mathrm{Ca}_{\mathrm{p}}$ and the ash percentage of the tibia, but strongly decreased the dry weight and total ash content of that bone. Dietary distribution of SM only did not modify the chick response to acidosis ; on the contrary, the mixture $D_{3}+S M$ decreased the deleterious effect of acidosis on bone development by about 30 p. 100 , and even elevated the ash percentage of the tibia to a higher value than that recorded in the non-acidotic birds.

\section{Introduction.}

L'acidose métabolique chronique entraîne chez les animaux de laboratoire une augmentation de mobilisation osseuse conduisant, chez l'adulte, à un tableau voisin de l'ostéoporose humaine (Barzel, 1976) : les concentrations plasmatiques de calcium et de phosphore sont peu ou pas affectées (en présence de parathormone et d'une source de vitamine D) tandis que l'excrétion urinaire de ces deux éléments croît (Cuisinier-Gleizes et al., 1967, 1975).

Les relations existant entre l'équilibre acido-basique et la balance calcique sont certainement complexes : cette balance dépend autant de l'absorption intestinale que de la mobilisation osseuse ou de l'excrétion rénale de calcium, elles-mêmes sous la dépendance de la parathormone (PTH), de la calcitonine et du 1,25-dihydroxycholécalciférol $\left(1,25-(O H)_{2} D_{3}\right)$. L'acidose métabolique peut affecter directement un des organes impliqués ou agir sur la synthèse et/ou la sécrétion des hormones citées.

Pour ce dernier point, le plus grand nombre d'études a porté sur une hypersécrétion éventuelle de PTH induite par l'acidose ; il semble, selon Coe ef al. (1975), que la 
quantité de PTH immunoréactive circulante s'accroisse effectivement chez l'homme en acidose métabolique chronique et ce, par l'intermédiaire probable de l'hypercalciurie. II a été également montré que l'acidose chronique induite par $\mathrm{NH}_{4} \mathrm{Cl}$ réduit la synthèse rénale de $1,25-(\mathrm{OH})_{2} D_{3}$ tant chez le poulet (Sauveur ef al., 1977) que chez le rat (Lee et al., 1977) privés de vitamine $D$.

II était donc intéressant d'étudier si un métabolite de la vitamine $D$ n'exigeant pas d'activité de la $25-(\mathrm{OH}) \mathrm{D}_{3}$-1-hydroxylase rénale permettrait ou non de surmonter les effets de l'acidose métabolique sur le métabolisme calcique. La source de métabolite actif utilisée a été le Solanum malacoxylon (SM) connu pour accroitre l'absorption intestinale du calcium même chez l'animal néphrectomisé (cf. revue de Vernejoul et al., 1978).

Sachant par ailleurs que le phosphore régule également l'activité de la $25-(\mathrm{OH}) \mathrm{D}_{3}$ 1-hydroxylase (Tanaka ef de Luca, 1973 ; Henry et Norman, 1974) et que le SM n'assure peut-être pas une phosphorémie normale au poulet rachitique (Cañas et al., 1977), deux apports alimentaires différents de phosphore ont été introduits dans le plan expérimental.

\section{Matériel et méthodes.}

Animaux : six cents poussins mâles Hubbard ont été répartis à l'âge de 2 jours dans 12 cases de $6 \mathrm{~m}^{2}$ renfermant 50 animaux chacune.

Régimes : douze aliments semi-synthétiques contenant tous 1 p. 100 de calcium ont été constitués (

— adjonction ou non de 2 p. 100 de chlorure d'ammonium ;

- contenu en phosphore fixé à 0,40 (niveau normal) ou 0,65 p. 100 (sous forme de phosphate bicalcique uniquement) :

- apport de vitamine $D_{3}$ seule (1500 Ul $/ \mathrm{kg}$ ), d'un mélange $D_{3}+S M$, ou de $S M$ seul.

Le SM a été incorporé à l'aliment sous forme de poudre de feuilles séchées; les quantités ont été fixées d'après les résultats de Procsal et al. (1976) estimant qu'un $\mathrm{kg}$ de feuilles sèches renferme de 10 à $60 \mathrm{mg}$ de produit actif; une valeur moyenne de $30 \mathrm{mg} / \mathrm{kg}$ a été retenue, soit approximativement une UI de produit actif $/ \mathrm{mg}$ de fevilles. Pour ajuster cet apport à celui de la vitamine $D_{3}$ (fixé à $1500 \mathrm{Ul} / \mathrm{kg}$ d'aliment), il a donc été incorporé $1,5 \mathrm{~g}$ de feuilles sèches de $S M / \mathrm{kg}$ d'aliment pour le lol ne recevant aucune autre source de vitamine $D$ et $0,8 \mathrm{~g}$ de $S M / \mathrm{kg}$ pour le lot recevant en complément $750 \mathrm{Ul} / \mathrm{kg}$ de vitamine $\mathrm{D}_{3}$.

Sachant que l'ingéré quotidien moyen d'un poulet évolue de 15 à $55 \mathrm{~g}$ entre la première ef la quatrième semaine d'âge, les concentrations alimentaires données ci-dessus ont dû conduire à des ingérés vitaminiques quotidiens évoluant de :

- 22 à $82 \mathrm{UI} / \mathrm{j}$ de vitamine $\mathrm{D}_{3}$ (lots $1,4,7$ et 10 ),

- 22 à $82 \mathrm{mg} / \mathrm{j}$ de feuilles sèches de SM (lots $3,6,9$ et 12),

- 11 à 40 Ul de $D_{3}+11$ à $40 \mathrm{mg} \mathrm{SM/j}$ (lots 2, 5, 8 et 11).

Mesures : Des prises de sang ont été effectuées sur 8 animaux par lot après une semaine d'élevage. Au cours de la deuxième semaine, l'acidose métabolique imposée a entraîné une très forte mortalité et l'expérience n'a pu être prolongée avec les six 


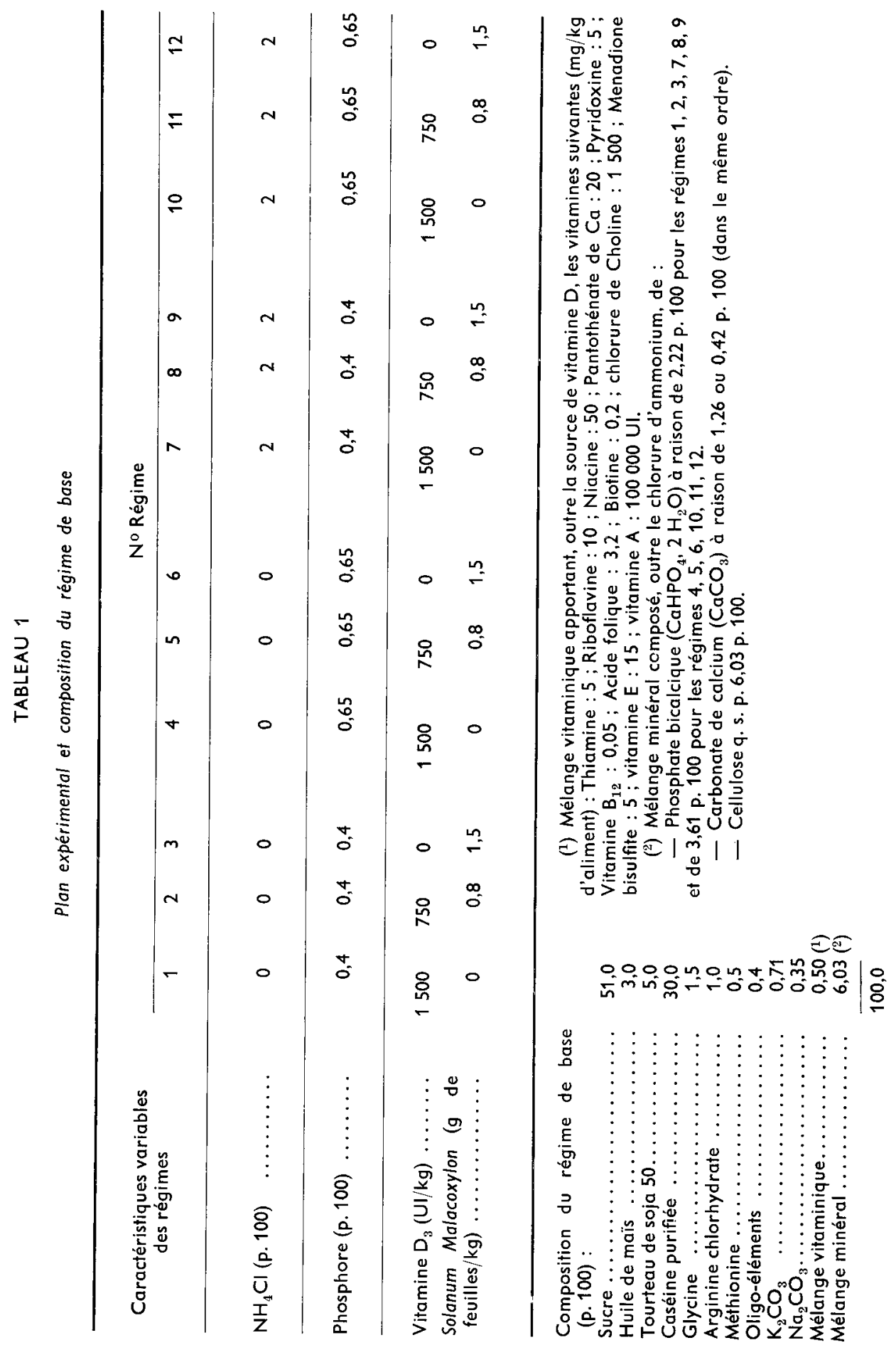


derniers lots; seuls les six premiers ont donc fait l'objet de prises de sang ultérieures (à 4 semaines d'âge).

Sur les échantillons de la première semaine ont été mesurés le $\mathrm{pH}$ et la $\mathrm{pCO}_{2}$ (microtonomètre d'Astrup Radiometer) ; la concentration des ions bicarbonate a été calculée par l'équation de Henderson et Hasselbach en prenant $p K^{\prime}=6,09$ (d'après Helbacka ef al., 1964) et la constante de solubilité du $\mathrm{CO}_{2}$ dans le plasma à $41^{\circ} \mathrm{C}$ égale à $0,028 \mathrm{mM} / 1 / \mathrm{mm} \mathrm{Hg}$. La chlorémie a été mesurée avec un chloromètre CMT 10 Radiometer. Sur tous les échantillons, la calcémie totale a été mesurée par spectrophotométrie de flamme (Eppendorf).

Le poids sec du tibia et sa teneur en cendres ont été déterminés sur un minimum de 5 échantillons de chaque lot à l'âge d'une semaine; il en a été de même à 4 semaines sur les six premiers lots seulement.

\section{Résultats.}

L'ensemble des analyses de variance (plans factoriels $2 \times 2 \times 3$ en première semaine et $2 \times 3$ en quatrième semaine) est donné au tableau 2, les résultats proprement dits étant illustrés par les figures 1 à 3.

TABLEAU 2

Analyse de variance des résulfats. Seuil de signification des valeurs de $F$

\begin{tabular}{|c|c|c|c|c|c|c|c|}
\hline \multirow[b]{2}{*}{ Paramètres } & \multicolumn{7}{|c|}{ Source de variation } \\
\hline & $\begin{array}{l}\text { Acidose } \\
\text { (A) }\end{array}$ & $\begin{array}{l}\text { Phos- } \\
\text { phore } \\
\text { (P) }\end{array}$ & $\begin{array}{l}\text { Source de } \\
\text { vitamine D }\end{array}$ & $A \times P$ & $P Y D$ & $A \succ D$ & $A \times P \times D$ \\
\hline \multicolumn{8}{|l|}{$\begin{array}{l}\text { Equilibre acido-basique d̀ } \\
1 \text { semaine (fig. } 1 \text { ) }\end{array}$} \\
\hline 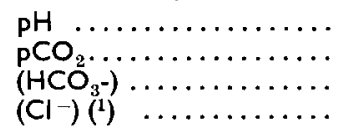 & $\begin{array}{l}* * * \\
* \\
* * * \\
* * *\end{array}$ & - & - & $\begin{array}{l}* \\
- \\
-\end{array}$ & - & $\begin{array}{l}- \\
-\end{array}$ & $\underline{-}$ \\
\hline \multicolumn{8}{|l|}{ Calcémie totale (fig. 2 ) } \\
\hline $\begin{array}{l}1 \text { semaine } \ldots \ldots \ldots \ldots \ldots \\
4 \text { semaines } \ldots \ldots \ldots \ldots \ldots\end{array}$ & $*$ & $\begin{array}{l}* * \\
*\end{array}$ & * & $*$ & - & 一 & - \\
\hline \multicolumn{8}{|l|}{ Etude du tibia (fig. 3) } \\
\hline 1 sem. $\left\{\begin{array}{l}\text { Poids sec } . . . . \\
\text { Cendres totales } \\
\text { Cendres p. } 100\end{array}\right.$ & $\begin{array}{l}* * * \\
* * * \\
*\end{array}$ & - & $\stackrel{\bullet}{*}$ & $\underset{* *}{-}$ & - & - & - \\
\hline $\begin{array}{l}4 \text { sem. } \\
\quad\left({ }^{2}\right)\end{array}\left\{\begin{array}{l}\text { Poids sec } \ldots . . . \\
\text { Cendres totales } \\
\text { Cendres p. } 100\end{array}\right.$ & & $\overline{-}$ & $\frac{-}{-}$ & & $\frac{-}{-}$ & & \\
\hline $\begin{array}{l}\text { Poids vif } \\
4 \text { semaines } \ldots \ldots \ldots \ldots \ldots\end{array}$ & & - & - & & - & & \\
\hline
\end{tabular}

- $0,10>p>0,05 ;\left(^{*}\right) p<0,05 ;\left(^{* *}\right) p<0.01 ;\left({ }^{* *}\right) \mathrm{p}<0.001 ;-$ valeur de $F<1$.

(1) La chlorémie n'a été dosée que dans les lots $1,3,7$ et 9.

(2) A 4 semaines d'âge, seuls subsistaient les lots 1 à 6 . 
1. Paramètres sanguins.

a) Equilibre acido-basique. - Les paramètres de l'équilibre acido-basique enregistrés à 1 semaine d'âge sont représentés sur la figure 1. L'administration de 2 p. 100 de chlorure d'ammonium entraîne une acidose métabolique extrêmement sévère se

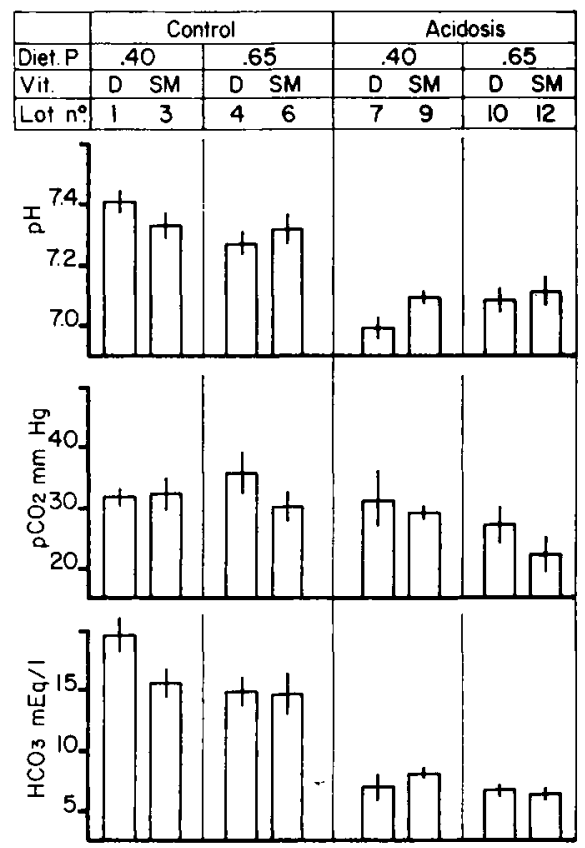

FIG. 1. - Paramètres de l'équilibre acido-basique sanguin chez les poulets âgés d'une semaine.

Valeurs de $\vec{X} \pm s / \sqrt{n}$ calculées sur 5 animaux par lot.

traduisant en moyenne par un pH de 7,07 et une bicarbémie abaissée jusqu'à $7 \mathrm{mEq} / \mathrm{l}$ (valeurs témoins respectives : 7,33 et 16 ) ( $p<0,001$ pour ces deux critères). La compensation respiratoire est modérée puisque la $\mathrm{PCO}_{2}$ ne diminue en moyenne que de 32 à $27 \mathrm{~mm} \mathrm{Hg}$ entre les lots témoins et ceux en acidose $(p<0,05)$.

Le taux de phosphore alimentaire paraît lui-même agir de façon discrète sur l'équilibre acido-basique ; l'apport le plus élevé (0,65 p. 100) entraîne par rapport au niveau témoin $(0,4$ p. 100) une légère acidose dans les lots ne recevant pas de chlorure d'ammonium ( $p<0,05$ pour la bicarbémie). En revanche, il n'intervient plus significativement sur ce paramètre chez les animaux acidotiques (lots 7 à 12). La source de vitamine $D$ utilisée (vitamine $D_{3}$ vs. $S M$ ) n'agit pas, en tant que telle, sur l'équilibre acido-basique.

La chlorémie n'a été mesurée que sur des échantillons en provenance des lots 1 , 3,7 et 9 (phosphore alimentaire égal à 0,4 p. 100) ; elle est en moyenne de $116,5 \mathrm{mEq} / \mathrm{l}$ dans les lots 7 ef 9 contre 102,7 dans les lots témoins 1 et 3 ( $p<0,001$ ) ce qui confirme l'importance de l'acidose hyperchlorémique induite. 
b) Calcémie. - Les valeurs de calcémie enregistrées à 1 et 4 semaines d'âge sont illustrées par la figure 2. Chez les animaux non acidotiques (lots 1 à 6) l'incorporation de $S M$ en substitution totale de vitamine $D_{3}$ entraîne une diminution de calcémie égale à 12 et $9 \mathrm{mg} / \mathrm{l}$ à 1 et 4 semaines respectivement $(p<0,05$ dans les deux cas) ; les lots 2 et 5 recevant un mélange de vitamine $D_{3}$ ef de $S M$ montrent des valeurs de calcémie intermédiaires non significativement différentes des valeurs extrêmes.

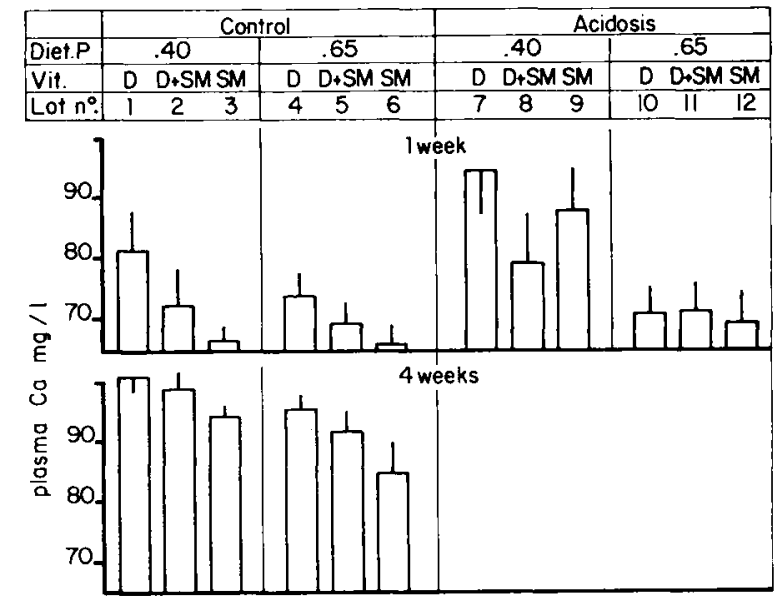

FIG. 2. - Calcémie totale des poulets à 1 et 4 semaines d'âge (les lots en acidose n'ont pu être maintenus au-delà d'une semaine). Valeurs de $\vec{X} \pm s / \sqrt{n}$ calculées sur 8 animaux par lot.

L'acidose métabolique en elle-même augmente légèrement la calcémie à une semaine lorsque l'apport alimentaire de phosphore est normal $(0,4$ p. 100) alors qu'elle n'agit pas en présence d'un taux de phosphore du régime égal à 0,65 p. 100 (interaction acidose $\times$ phosphore $: p<0,05$ à 1 semaine). Aucune conclusion certaine ne peut être tirée quant à l'effet de la source vitaminique sur la calcémie des animaux en acidose; il semble cependant que, en présence d'acidose, le SM diminue moins la calcémie par rapport à la vitamine $D_{3}$ qu'il ne le fait chez les animaux non acidotiques (comparer lots 9 vs. 7 et 3 vs. 1 ou lots 12 vs. 10 et 6 vs. 4).

Le fait d'augmenter la teneur de l'aliment en phosphore de 0,4 à 0,65 p. 100 induit une baisse de calcémie, quels que soient les autres traitements $(p<0,01$ pour les deux âges éłudiés).

\section{Croissance et minéralisation du tibia.}

La figure 3 représente aux deux âges étudiés les résultats enregistrés pour le poids sec du tibia, la quantité totale de cendres et la teneur en cendres exprimée en p. 100 du poids sec.

L'acidose métabolique entraîne une réduction importante du poids du tibia ( $p<0,001$ à 1 semaine) quel que soit l'apport alimentaire de phosphore ; la teneur en cendres est elle-même légèrement réduite par l'acidose lorsque l'apport de phos- 
phore est limité à 0,4 p. 100 (interaction acidose $\times$ phosphore : $p<0,01$ ). II en résulte une baisse de la quantité totale de cendres du tibia entre les lots témoins et ceux.en acidose.

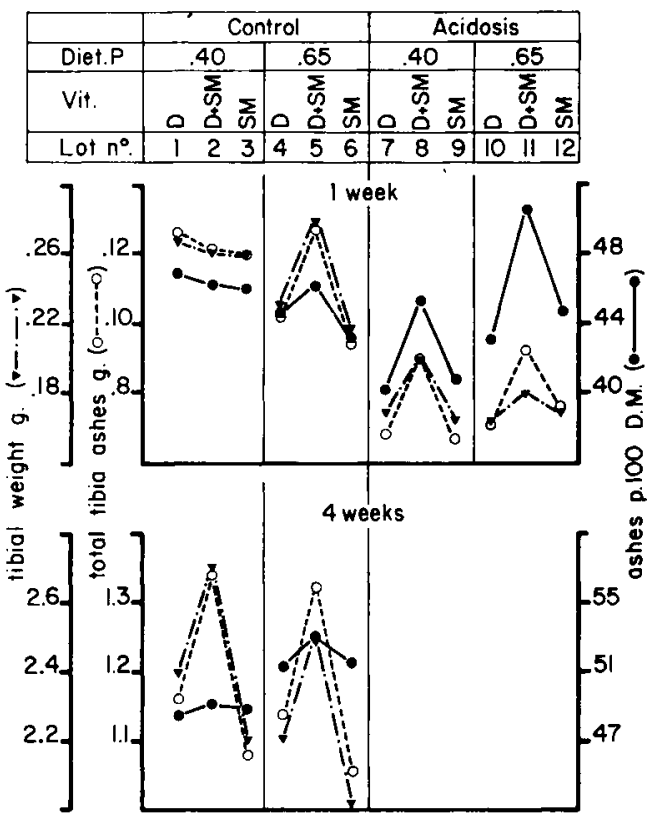

FIG. 3. - Croissance et minéralisation du fibia à 1 ef 4 semaines d'âge. Valeurs moyennes du poids sec du tibia (4- - $)$, du poids total de cendres $(0--0)$ et du pourcentage de cendres par rapport au poids sec (•-) calculées sur 5 animaux par lot.

L'apport de phosphore alimentaire le plus élevé (0,65 p. 100) assure une minéralisation plus élevée du tibia que le niveau témoin ( 0,4 p. 100). Pour les lots sans acidose, cet effet n'est visible que durant la 4 e semaine $(p<0,001)$ alors qu'en présence $d^{\prime} u n e$ acidose, cet effet bénéfique du phosphore apparaît dès la première semaine $(p<0,01)$. A l'opposé, le taux de phosphore de l'aliment n'agit significativement ni sur le poids sec du tibia ni sur les valeurs de cendres totales.

Si l'on considère seulement les lots 1 à 3 en fin de première semaine (lots témoins avec apport de phosphore normal et absence d'acidose), il n'existe aucun effet de la source vitaminique sur la croissance du tibia ou sa teneur en cendres. A l'opposé, dans les 9 lots suivants, le mélange $D_{3}+S M$ conduit à des valeurs plus élevées que la $D_{3}$ seule ou le $S M$ seul, surtout pour la teneur en cendres $(p<0,01)$. II en résulte en particulier que, sous acidose, ce mélange $D_{3}+S M$ permet d'atteindre des teneurs en cendres du tibia égales ou supérieures à celles enregistrées dans les lots non acidotiques correspondants ; pour les cendres totales du tibia, l'action bénéfique du mélange $\mathrm{D}_{3}+\mathrm{SM}$ est cependant insuffisante pour compenser totalement l'effet négatif de l'acidose (comparer lots 8 vs. 1 ou 11 vs. 4 ).

A 4 semaines d'âge, la combinaison $D_{3}+S M$ paraît également assurer la meil- 
leure croissance osseuse mais les écarts enregistrés ne sont pas statistiquement significatifs. II en est de même pour le poids vif à 4 semaines dont les valeurs sont en moyenne de 598, 665 et $607 \mathrm{~g}$ pour les lots recevant respectivement la vitamine $D_{3}$ seule, le mélange $D_{3}+S M$, ef le $S M$ seul (différences non significatives au seuil de probabilité 0,05).

\section{Discussion.}

Le présent essai a été réalisé d'une part pour étudier si le Solanum Malacoxylon pouvait être substitué en totalité à la vitamine $D_{3}$ chez le poulet en croissance, d'autre part pour voir s'il compensait certains troubles du métabolisme calcique induits par une acidose métabolique. Comple tenu de la forme d'incorporation de SM utilisée (poudre de feuilles séchées), il subsiste évidemment une certaine incertitude quant aux doses de composé actif administré (revoir matériel et méthodes).

\section{Action du SM chez le poulet non acidique.}

Les résultats obtenus avec les lots 1 à 3 du présent essai montrent que l'incorporation à l'aliment de $1,5 \mathrm{~g}$ de feuilles sèches de $S M / \mathrm{kg}$ d'aliment conduit, en présence d'un apport normal de phosphore $(0,4 p .100)$, à des résultats très voisins de ceux donnés par $1500 \mathrm{UI} / \mathrm{kg}$ de vitamine $D_{3}$ en ce qui concerne le poids vif à 4 semaines et la teneur en cendres du tibia. A l'opposé, la calcémie est réduite par l'apport du SM comparé à celui de $D_{3}$.

Peu de travaux ont été réalisés dans des conditions exactement similaires pouvant permettre une comparaison ; selon Cañas ef al. (1977) la distribution quotidienne d'un extrait aqueux de $250 \mathrm{mg}$ de SM/poulet après 2 semaines de rachitisme préalable, normalise la calcémie et le contenu en cendres de l'os mais ne restaure ni la phosphorémie, ni un poids vif identique à ceux des témoins non rachitiques. S'il n'est pas possible de discuter ici des valeurs de phosphatémie (les dosages de $P$ minéral n'ayant pu être réalisés par suite d'une erreur de manipulation), il est clair que la dose de SM distribuée, bien qu'inférieure à celle citée ci-dessus, assure une croissance corporelle normale.

L'action du SM sur l'os est controversée et varie peut-être avec la durée d'administration du produit (de Vernejoul et al., 1978). Selon Puche et al. (1976) la distribution chronique de SM à des rats normalement approvisionnés en calcium réduit l'accrétion et augmente la résorption osseuse mesurée par cinétique du ${ }^{45} \mathrm{Ca}$; cependant, l'excrétion urinaire d'hydroxyproline diminue et les auteurs concluent à une élévation du taux de synthèse du tissu osseux. A l'état aigu ou in vitro, l'action la plus nette du SM est l'accroissement de résorption osseuse (Puche et Locatto, 1974 ; Simonite ef al., 1976).

Dans les conditions chroniques du présent essai, le SM assure chez le poulet (non acidotique) une croissance et une teneur en cendres de l'os normales ; l'abaissement de calcémie observé peut cependant faire penser à une réduction modeste de mobilisation osseuse ou à une augmentation d'excrétion urinaire du calcium (Puche ef al., 1976). 
2. Rôle du niveau d'apport de phosphore chez le poulet non acidotique.

La légère tendance à l'acidose induite par le taux de phosphore alimentaire le plus élevé s'explique peut-être par la composition même des régimes puisque, afin de conserver un contenu calcique constant, ceux riches en phosphore (apporté par du phosphore bicalcique) contiennent moins de carbonate de calcium (tabl. 1).

Il est connu que le besoin en phosphore du poulet pour obtenir une teneur en cendres de l'os maximale est supérieur à celui estimé par la seule croissance corporelle; Fritz (1974) estime ainsi le besoin en phosphore total à 0,5 ou 0,65 p. 100 selon qu'il recherche la croissance ou l'ossification maximales. Les résultats présents confirment ce fait puisque, en l'absence d'acidose, la teneur en cendres du tibia à 4 semaines croît de 3 p. 100 avec l'apport de phosphore le plus élevé.

On sait par ailleurs qu'un régime riche en phosphore réduit la synthèse rénale de $1,25-(\mathrm{OH})_{2} \mathrm{D}_{3}$ (Larkins ef al., 1973). Cette régulation peut être impliquée dans la diminution de calcémie observée ici avec l'apport de phosphore le plus élevé quel que soit l'âge ou l'état d'équilibre acido-basique. Il faut cependant noter que l'apport de SM ne permet pas de compenser cette action dépressive du phosphore sur la calcémie ce qui conduit à envisager également une action freinatrice directe d'un léger excès de phosphate minéral sur l'absorption intestinale du calcium, via la formation de composés insolubles (Hurwitz et Bar, 1971).

\section{Interaction acidose métabolique $\times S M$.}

Il convient d'abord de souligner la gravité de l'acidose métabolique imposée dès la naissance et qui n'a été que peu compensée par les animaux, probablement du fait d'insuffisances rénales. Ceci s'est traduit par une forte mortalité à partir de la deuxième semaine ef limite donc la portée des résultats obtenus sur les effets conjoints de l'acidose et de la source de vitamine $D$.

Compte tenu de ces restrictions, l'acidose métabolique entraîne à une semaine d'âge une légère augmentation de la calcémie ; cette augmentation disparaît dans les jours qui suivent (résultats non rapportés ici), probablement en liaison avec la mise en place d'une hypercalciurie caractéristique des états d'acidose (Cuisinier-Gleizes ef al., 1967, 1975).

Au niveau osseux, l'acidose métabolique est connue pour accroître les phénomènes de résorption sans qu'il soit définitivement établi si une modification des populations cellulaires de l'os est impliquée ou s'il ne s'agit que d'un phénomène chimique ; certains auteurs parlent en outre d'une diminution d'accrétion alors que d'autres estiment ce paramètre inchangé (voir la revue de Barzel, 1976). Les auteurs sont en revanche unanimes pour affirmer que l'acidose métabolique, sans affecter le volume osseux, en réduit le poids sec, les cendres totales et le contenu en calcium tandis que le rapport cendres/matrice reste normal.

Les résultats présents sont en accord avec ces faits bien qu'ils aient été obtenus sur des animaux très jeunes : sil l'on ne considère que les lots recevant de la vitamine $D_{3}$ seule, le poids sec de l'os est en effet réduit de 33 p. 100 par l'acidose alors que la teneur en cendres (rapportée au poids sec) ne varie que de 9 p. 100.

La source de vitamine $D$ utilisée modifie la réponse osseuse à l'acidose el ceci de façon assez surprenante ; si le SM seul conduit en effet à des valeurs tout à fait compa- 
rables à celles données par la $D_{3}$ seule, le mélange $D_{3}+S M$ compense en partie les troubles osseux induits par l'acidose ef permet d'obtenir une valeur de cendres totales du tibia inférieures de seulement 27 p. 100 à celle des lots correspondants non acidotiques (contre 40 p. 100 dans les lots avec $D_{3}$ ). L'effet est encore plus net pour la teneur en cendres rapportée au poids sec pour laquelle le mélange $D_{3}+S M$ permet aux lots en acidose de dépasser les lots témoins correspondants (augmentation relative de 3,3 p. 100) alors qu'en présence de $D_{3}$ seule ce paramètre, comme on l'a déjà dit, est abaissé de 9 p. 100 par l'acidose.

II a été montré que l'acidose métabolique réduit les capacités de synthèse rénale de $1,25-(\mathrm{OH})_{2} \mathrm{D}_{3}$ chez l'animal carencé en vitamine D (Sauveur ef al., 1977 ; Lee et al., 1977) mais il n'est pas démontré qu'il en soił de même chez l'animal recevant normalement de la vitamine $D$. On ignore par ailleurs si la réponse osseuse à l'acidose passe ou non par les métabolites du cholécalciférol. Dans ce contexte, le fait qu'un mélange $\mathrm{D}_{3}+\mathrm{SM}$ permette une meilleure résistance osseuse à l'acidose que le SM seul est difficile à interpréter mais peut indiquer une voie thérapeutique intéressante dans certaines ostéopathies du poulet liées à l'équilibre acido-basique (Sauveur et Mongin, 1978). Un résultat de Leach et Simons (1976) est instructif en ce sens puisque ces auteurs ont pu démontrer une action préventive du 1,25-(OH) ${ }_{2} \mathrm{D}_{3}$ vis-à-vis de la dyschondroplasie tibiale, action qui disparaissait lorsque la dose administrée étaił multipliée par deux.

Reçu en mars 1979.

Accepté en juin 1979.

Remerciements. - L'auteur adresse ses sincères remerciements à B. J. Carrillo (CICV, INRA, Castelar, Argentina) pour la fourniture de Solanum Malacoxylon.

\section{Références}

BARZEL U. S., 1976. Acid-induced osteoporosis : an experimental model of human osteoporosis. Calcif. Tiss. Res., 21, (suppl.), 417-422.

CAÑAS F. M., ORTIZ O. E., ASTEGGIANO C. A., PEREIRA R. D., 1977. Effects of Solanum MalacoxyIon extract on rachitic chicks. Calcif. Tiss. Res., 23, 297-301.

COE F. L., FIRPO J. J., don HOLLANDSWORTH L., SEGIL L., CANTERBURY J. M., REISS E., 1975. Effect of acute and chronic metabolic acidosis on serum immunoreactive parathyroid hormone in man. Kidney Int., 8, 262-273.

CUISINIER-GLEIZES P., MATHIEU H., ROYER P., 1967. Effet d'une surcharge acide sur l'équilibre phosphocalcique du rat parathyroïdectomisé et du rat normal. Rev. franç. Ełudes clin. biol., 12, 566-573.

CUISINIER-GLEIZES P., BENEST D., GEORGE A., THOMASSET M., 1975. Thyroparathyroid glands, bone and acid-base homeostasis. Proc. Xlth europ. Symp. Calcif. Tiss. (Abstr.), 70.

FRITZ J. C., 1974. Phosphorus requirements of poultry. Proc. Maryland Nutr. Conf., 96-102.

HELBACKA N. V. L., CASTERLINE J. L., SMITH C. J., SHAFFNER C. S., 1964. Investigation of plasma carbonic acid $\mathrm{PK}^{\prime}$ of the chicken. Poultry Sci., 43, 138-144.

HENRY H., NORMAN A. W., 1974. Modulation in vitro of chick kidney 25-hydroxycholecalciferol-1hydroxylase. Fed. Proc., 33, 679.

HURWITZ S., BAR A., 1971. Calcium and phosphorus interrelationships in the intestine of the fowl. J. Nutr., 101, 677-686.

LARKINS R. G., Mac AULAY S. J., COLSTON K. W., EVANS I. M. A., GALANTE L. S., Mac INTYRE I. 1973. Regulation of vitamin D metabolism without parathyroid hormone. Lancet, ii, 289-291. 
LEACH R. M., SIMONS P., 1976. Communication personnelle.

LEE S. W., RUSSEL J., AVIOLI L. V., 1977. 25-hydroxycholecalciferol to 1.25-dihydroxycholecalciferol : Conversion impaired by systemic metabolic acidosis. Science, 195, 994-996.

PROCSAL D. A., HENRY H. L., HENDRICKSON T., NORMAN A. W., 1976. 1 $\alpha, 25-d i h y d r o x y-$ vitamin $D_{3}$-like component present in the plant Solanum Malacoxylon. Endocrinology, 99, 437 444.

PUCHE R. C., LOCATTO M. E., 1974. Effects of Solonum Malacoxylon on embryonic bone in vifro and on isolated Mitochondria. Calcif. Tiss. Res., 16, 219-226.

PUCHE R. C., LOCATTO M. E., FERRETTI J. L., FERNANDEZ M. C., ORSATTI H. B., VALENTI J. L., 1976. The effects of long term feeding of Solonum Glaucophyllum to growing rats on $\mathrm{Ca}, \mathrm{Mg}$, $P$ and bone metabolism. Calcif. Tiss. Res., 20, 105-119.

SAUVEUR B., GARABEDIAN M., FELLOT C., MONGIN P., BALSAN S., 1977. The effect of induced metabolic acidosis on vitamin $D_{3}$ metabolism in rachitic chicks. Calcif. Tiss. Res., 23, 121-124.

SAUVEUR B., MONGIN P., 1978. Tibial dyschondroplasia, a cartilage abnormality in poultry. Ann. Biol. anim. Bioch. Biophys., 18, 87-98.

SIMONITE J. P., MORRIS K. L. M., COLLINS J. C., 1976. Induction of bone resorption in vitro by an extract of Solanum Malacoxylon. J. Endocrinol., 68, 18P-19P.

TANAKA Y., DE LUCA H. F., 1973. The control of 25-hydroxyvitamin D metabolism by inorganic phosphorus. Arch. Biochem. Biophys., 154, 566-574.

DE VERNEJOUL M. C., MAUTALEN C. A., MIRAVET L., 1978. Le Solanum Malacoxylon : de la plante toxique à l'agent thérapeutique. Nouv. Presse méd., 7, 1941-1943. 